UCLA School of Engineering and Applied Science

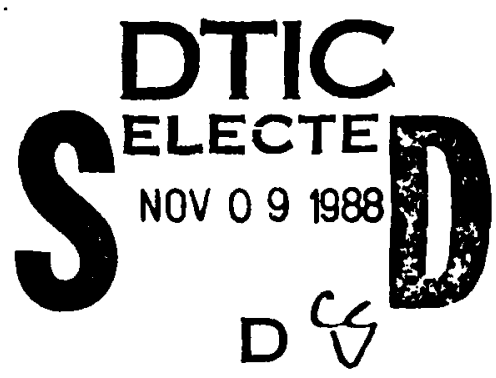

Reciprocal Theorem of Residual Stress

and Inelastic Strain

The report was sponsored by the Office of Naval Research Through contract N00014-86-K0153

\begin{tabular}{c}
\hline DISTRIBUTION STATEMENT A \\
$\begin{array}{c}\text { Approved tor public releces } \\
\text { Distritution Ualimitgd }\end{array}$
\end{tabular}

T.H.Lin

S.R.Lin

X.Q.Wu

X.Y.Chen 


\section{Reciprocal Theorem of Residual Stress \\ and Inelastic Strain}

T.H. Lin ${ }^{*}$, S.R. Lin ${ }^{* *}$, X.Q. Wu ${ }^{*}$, and Q.Y. Chen ${ }^{*}$

\section{Introduction:}

We consider a body to have an initial stress field. $f_{i j}$; When the body is loaded beyond the elastic range, plastic strain $e_{i j}^{p}$ occurs in some part of the body. If the body is then unloaded, the plastic strain remains and causes a residual stress field. $\tau_{i j}^{R}$, Now consider the load applied again. This applied load causes a stress $\tilde{\tau}_{\mathrm{ij}}^{\mathrm{A}}$. There is no increase of plastic strain during this process of unloading and reloading. Now the stress field is the sum of the initial, the residual and the applied stress

$$
\tau_{i j}=\tau_{i j}^{I}+\tau_{i j}^{R}+\tau_{i j}^{A}
$$

As shown by Lin, 1968, using this analogy between plastic strain and applied force, we can find $\tau_{i j}^{R}$ in terms of $e_{i j}^{P}$ in the body. As the load is increased, $\tau_{i j}^{A}$ increases. Then from the incremental stress strain relation of the material, the incremental plastic strains in different parts of the body are found (Lin, 1968). In this inelastic analysis, the relation between the plastic strain and the residual stress is needed. Plastic strain is one type of inelastic strain. Hence the reciprocal relation of inelastic strain and residual stress is very useful in numerical inelastic analyses.

\footnotetext{
* Department of Civil Engineering University of California, Los Angeles, Los Angeles, CA 90024-1593

** The Aerospace Corporation, Structural Technology Office, El Segundo, CA 90045
} 


\section{Derivation of the Reciprocal Relation for Inelastic Bodies:}

Referring to a set of rectangular coordinates $\left(x_{1}, x_{2}, x_{3}\right)$, the equilibrium condition is given as

$$
\tau_{i j, j}+F_{i}=0
$$

in the interior of the volume $\mathrm{V}$, and

$$
S_{i}=\tau_{i j} v_{j}
$$

on the boundary $\Gamma$ where $\tau_{1 j}$ is the stress component. $F_{1}$ the body force per unit volume along $x_{i}$-direction. $S_{i}$ is the traction per unit area along $x_{i}$-axis and $v_{j}$ is the direction cosine of the exterior normal of the boundary with $x_{j}$-axis. The repetition of the subscript denotes summation from one to three. and the subscript after comma denotes differentiation with respect to that axis. Multiplying (2) and (3) by a virtual displacement $u_{i}^{*}$ and integrating yields

$$
\begin{aligned}
\int F_{i} u_{i}^{*} d v+\int S_{i} u_{i}^{*} d \Gamma & =-\int \tau_{i j, j} u_{i}^{*} d v+\int \tau_{i j} v_{j} u_{i}^{*} d \Gamma \\
& =\int \tau_{i j} u_{i, j}^{*} d v=\int \tau_{i j} e_{i j}^{*} d v
\end{aligned}
$$

The stress-strain relation of an elastic body may be written as

$$
\tau_{\mathrm{ij}}=c_{\mathrm{ijkl}} \mathrm{e}_{\mathrm{kJ}}
$$

where $c_{i j k l}$ is the elastic constant.

$$
\int \tau_{i j} e_{i j}^{*} d v=\int c_{i j k l} e_{k l} e_{i j}^{*} d v=\int \tau_{k l}^{*} e_{k l} d v
$$

From (4) and (6)

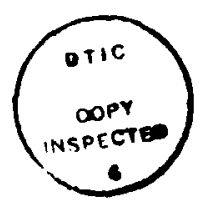

$$
\int F_{i} u_{i}^{*} d v+\int S_{i} u_{i}^{*} d \Gamma=\int F_{i}^{*} u_{i} d \Gamma+\int S_{i}^{*} u_{i} d \Gamma
$$

This is Betty's reciprocal theorem for elastic bodies.

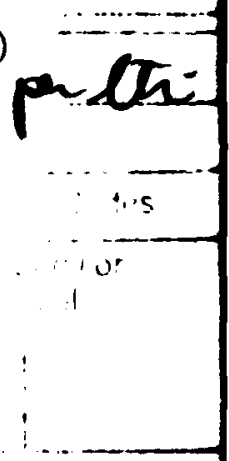


Now consider the presence of inelastic strain $e_{i j}^{\prime \prime}$ which may be a combination of plastic strain. creep strain and thermal strain. The total strain $e_{i j}$ is composed of the elastic strain $e_{1 j}^{\prime}$ and inelastic strain $e_{1 j}^{\prime \prime}$

$$
\begin{aligned}
& e_{i j}^{\prime}=e_{i j}-e_{i j}^{\prime \prime} \\
& \tau_{i j}=c_{i j k l}\left(e_{k l}-e_{k l}^{\prime \prime}\right)
\end{aligned}
$$

Substituting this into (2) and (3) yields

$$
\begin{aligned}
& c_{i j k l}\left(e_{k l, j}-e_{k l, j}^{\prime \prime}\right)+F_{i}=0 \\
& S_{i}=c_{i j k !}\left(e_{k l}-e_{k !}^{\prime \prime}\right) v_{j}
\end{aligned}
$$

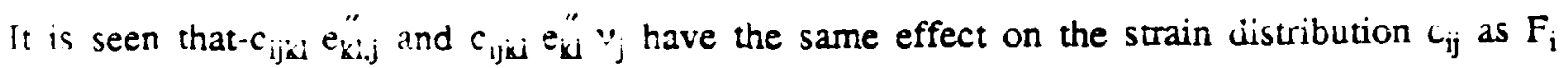
and $S_{i}$ respectively and hence they are denoted respectively as $\underline{F}_{i}$ and $\underline{S}_{i}$ ( $\left.\operatorname{Lin}, 1968\right)$.

Now consider an inelastic strain $e_{i j}^{\prime I}$ in region $I$ giving $\underline{F}_{i}^{I}$ and $\underline{S}_{i}^{I}$. The displacement, strain and stress in another region II caused by $e_{i j}^{\prime \mathcal{I}}$ are denoted by $u_{i}^{I I, I} e_{i j}^{I I, I}$ and $\tau_{i j}^{I I, I}$ respectively as shown in Fig. 1. 

Applying Betty's Reciprocal Theorem (7) to Figs. 1 and 2 gives

$$
\begin{aligned}
& \int_{v^{l}} \underline{F}_{i}^{I} u_{i}^{l, I I} d v+\int_{\Gamma^{I}} \underline{S}_{i}^{l} u_{i}^{l, I I} d \Gamma \\
& =\int_{v^{\mathrm{a}}} \underline{F}_{i}^{I I} u_{i}^{I l, I} d v+\int_{\Gamma^{\mathrm{a}}} \underline{S}_{i}^{I I} u_{i}^{I I, I} d \Gamma
\end{aligned}
$$

Replacing $\underline{F}_{j}$ by $-c_{i j k l} e_{k l j}^{\prime \prime}$ and $\underline{S}_{j}$ by $c_{i j k l} e_{k l}^{\prime \prime} v_{j}$ yields

$$
\begin{aligned}
& -\int_{v^{1}} c_{i j k l} e_{k l, j}^{\prime I I} u_{i}^{I, I I} d v+\int_{\Gamma} c_{i j k l} e_{k\rfloor}^{\prime I} v_{j} u_{i}^{I, I I} d \Gamma \\
& =-\int_{v^{\mathbb{I}}} c_{i j k l} e_{k l, j}^{\prime \prime I I} u_{i}^{I I, I} d v+\int_{\Gamma^{\prime \prime}} c_{i j k\rfloor} e_{k J}^{\prime \prime I I} v_{j} u_{i}^{I, I I} d \Gamma
\end{aligned}
$$

Using divergence theorem and noting $c_{i j k l}=c_{i j k l} E q(11)$ becomes

$$
\begin{aligned}
& \int_{v^{\prime}} c_{i j k l} e_{k l}^{\prime \prime I} e_{i j}^{I, I I} d v=\int_{v^{\prime \prime}} c_{i j k l} e_{k l}^{\prime \prime I I} e_{i j}^{I, I I} d v \\
& \int_{v^{l}} \tau_{k l}^{I I I} e_{k l}^{\prime \prime I} d v=\int_{v^{I I}} \tau_{k j}^{I I, I} e_{k l}^{\prime I I} d v
\end{aligned}
$$

For the case $v^{I}=v^{I I}$ and $e_{k l}^{\prime I} \neq e_{k l}^{\prime I I}$, the relation (12) still holds.

$\tau_{\mathrm{ij}}^{\mathrm{I}, \mathrm{II}}$ is the stress in region I causes by inelastic strain $\mathrm{e}_{\mathrm{ij}}^{\prime \prime I}$ in region II and $\tau_{\mathrm{ij}}^{\mathrm{II}, \mathrm{I}}$ is the stress in region II caused by inelastic strain $\mathrm{e}_{\mathrm{ij}}$ 'I in region I. If regions I and II have no overlap, the stress $\tau_{\mathrm{kl}}^{\mathrm{III}}$ and $\tau_{\mathrm{kl}}^{\mathrm{II}, \mathrm{I}}$ are residual stresses. If there is overlap as shown in Fig. 3 . The residual stress in the overlapped region, denoted hy $v^{I} \cap v^{I I}$, is then

$$
\begin{aligned}
& \tau_{k l_{l}^{I I}}^{I I I}=\tau_{k l}^{I I I}-c_{k 1 m n} e_{m n}^{\prime I I} \\
& \tau_{k l_{\rho}^{I I}}^{I I I I}=\tau_{k l}^{I I I}-c_{k l m n} e_{m n}^{\prime I}
\end{aligned}
$$

This gives

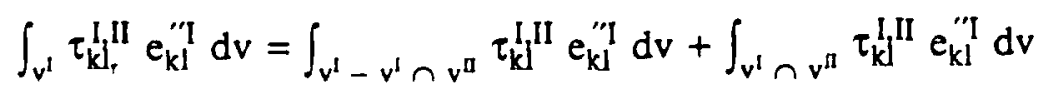




$$
\begin{aligned}
& -\int_{v^{1} \cap v^{I I}} c_{i j k l} e_{k !}^{\prime I I} e_{i j}^{\prime I} d v
\end{aligned}
$$

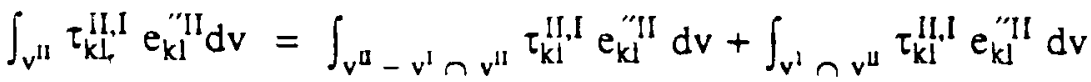

$$
\begin{aligned}
& -\int_{v^{1} \cap v^{I I}} c_{i j k l} e_{k d}^{\prime \prime I} e_{i j}^{\prime I I} d v
\end{aligned}
$$

From (12) to (15)

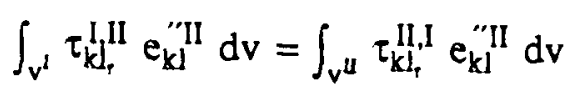

This gives the reciprocal relation for inelastic strain and residual stress in solids.

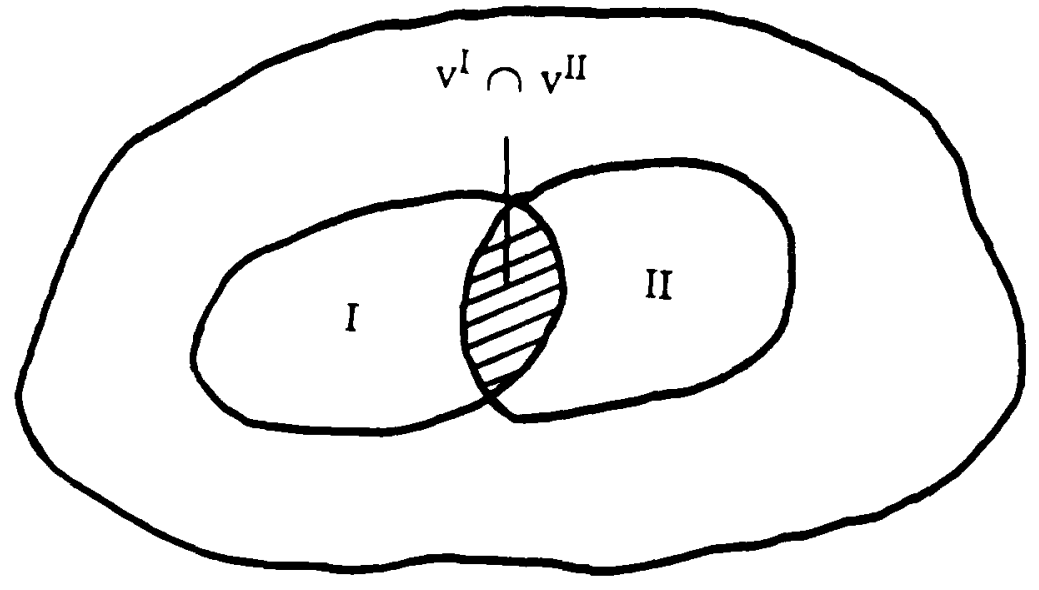

Fig. 3. $v^{I}$ and $v^{I I}$ have an overlap $v^{I} \cap v^{2}$ 


\section{Application to Fatigue Band Calculations:}

A face-centered-cubic crystal has four slip planes, on each of which there are three slip directions giving tweive slip systems. Slip in a slip system depends on the resolved shear stress and is independent of the normal stress on the sliding plane (Taylor, 1938). Let us consider a most favorably oriented crystal in a F.C.C. polycrystal. When this polycrystal is loaded. slip may occur in some region of this crystal. After unloading, this slip remains and causes a residual stress. Imagine that the polycrystal is reloaded. This resolved shear stress in this crystal can be expressed as the sum of the initial resolved shear stress $\tau^{\mathbb{I}}$, the resolved shear stress due to the applied loading $\tau^{A}$ and the residual shear stress $\tau^{\mathbb{R}}$. If this resolved shear stress reaches the critical shear stress $\tau^{\mathfrak{c}}$, i.e.

$$
\tau=\tau^{\alpha}+\tau^{A}+\tau^{R}=\tau^{\mathfrak{c}}
$$

in some region, this region will start or continue to slide.

We consider three thin slices in a most favorably oriented crystal at the free surface of the polycrystal as shown in Fig.4 ( $\mathrm{Lin}, 1974)$. An initial tensile strain $\mathrm{e}_{\alpha \alpha}^{I}$ is assumed to exist in the slice $R$. This $\mathrm{e}_{\alpha \alpha}^{l}$ causes an initial compressive stress in $R$, a positive resolved shear stress $\tau_{\alpha \beta}^{1}$ in $P$ and a negative $\tau_{\alpha \beta}^{\mathrm{I}}$ in $Q$ (Lin and Lin, 1988). A cyclic loading of tension and compression on the polycrystal causes altemate sliding in $P$ and $Q$ causing the growth of an extrusion or an intrusion ( $\mathrm{Lin}, 1977$, Lin and Ito, 1969). An extrusion causes a tensile strain and hence a tensile stress along extrusion direction. This can cause the resolved shear stress in a second slip system to slide in $R$. This secondary slip system can slide concurrently with this primary slip system in $P$ and $Q$. Let $\alpha \beta$ denote the primary slip system and $\xi \eta$, the second slip system. The plastic strains caused by slip in these two systems are denoted by $e_{\alpha \beta}^{P}$ and $e_{5 \eta \eta}^{P}$ respectively. The resolved shear stress $\tau_{\alpha \beta}^{R}(x)$ in $\alpha \beta$-system at point $x$ caused by

a unit $e_{\alpha \beta}^{p}$ at $\bar{x}$ in a differential volume $d v$ is expressed as $G(x, \alpha \beta ; \bar{x}, \alpha \beta) e_{\alpha \beta}^{P} d v$. If $e_{\xi \eta}^{p}$ also occurs, we can write 


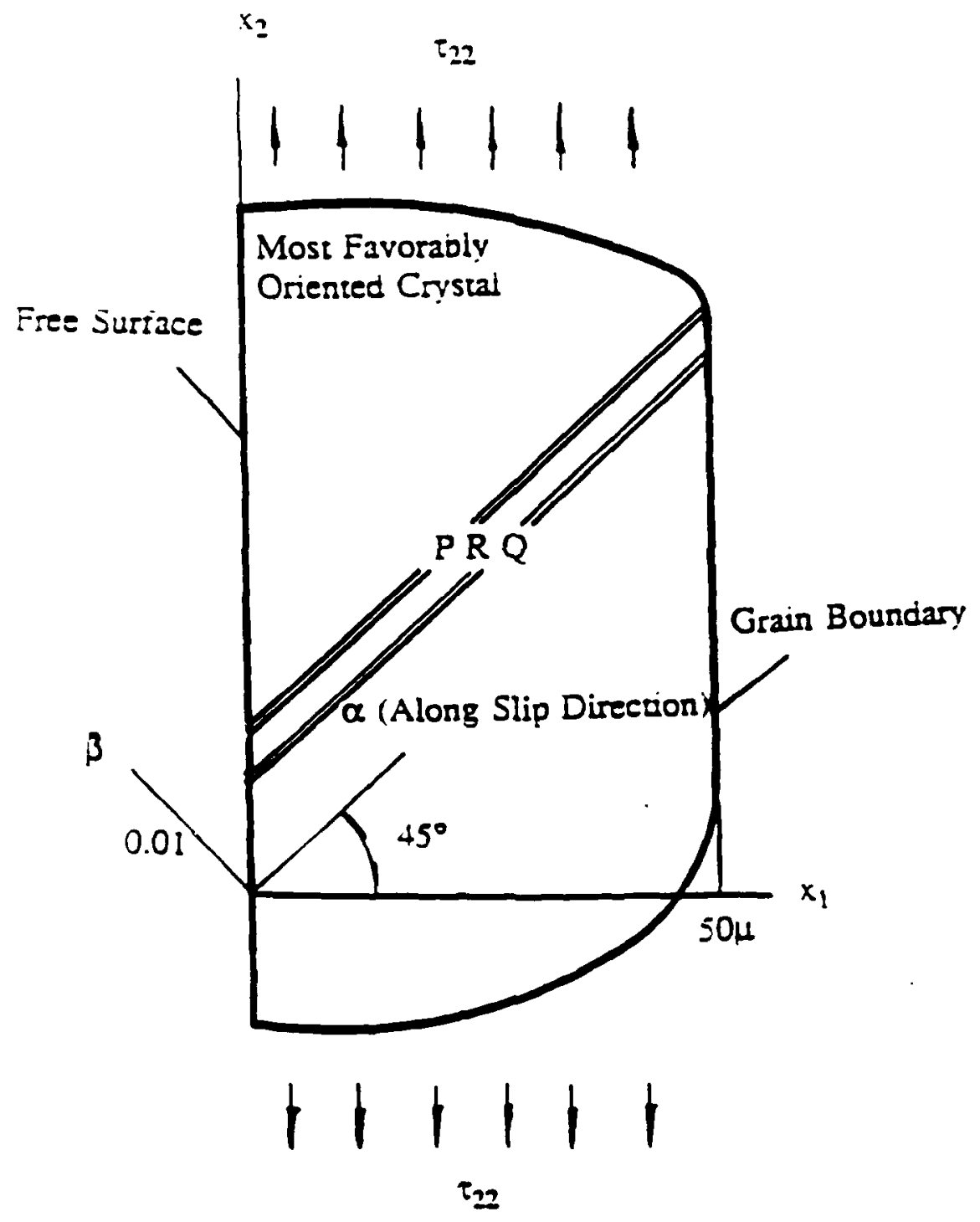

Fig.4 Most Favorable Oriented

Crystal at Free Surface 


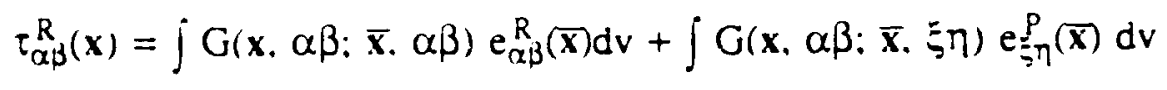

The two integrals are integrated over the regions with plastic strain. Similarly we can write

$$
\tau_{\xi \eta}^{R}(\mathbf{x})=\int G(x, \bar{\xi} \eta ; \bar{x}, \alpha \beta) e_{\alpha \beta}^{p}(\bar{x}) d v+\int G(x, \xi \eta ; \bar{x}, \xi \eta) e_{\xi \eta}^{p}(\bar{x}) d v
$$

In numerical calculation. the thin slices $P, Q, R$ are divided into grids. In each grid. the $e_{\alpha \beta}^{P}$ or $e_{F \eta}^{P}$ is generally assumed to be constant or linear. The residual resolved shear stress due to plastic strains in the nth grid is written as

$$
\tau_{\alpha \beta}^{R}(x)=G(x, \alpha \beta ; n, \alpha \beta) e_{\alpha \beta_{n} v_{n}}^{p}+G(x, \alpha \beta ; n, \bar{\zeta} \eta) e_{\xi \eta}^{p} v_{n}
$$

where

$$
G(x, \alpha \beta ; n, \alpha \beta)=\frac{\int_{v_{s}} G(x, \alpha \beta ; \bar{x}, \alpha \beta) e_{\alpha \beta}^{p} d v}{\int_{v_{a}} e_{\alpha \beta}^{p}(\bar{x}) d v}
$$

and $e_{\alpha \beta_{n}}^{p}$ is the average $e_{\alpha \beta}^{p}$ in the nth grid. Now we consider the residual stress in the mth grid corresponding to an assumed plastic strain distribution in the grid. As mentioned before, this distribution is generally assumed to be constant or linear. Multiplying (21) by $e_{\alpha \beta}^{p} d v$ in this grid and integrate

$$
\begin{aligned}
& \int_{v_{m}} \tau_{\alpha \beta}^{R}(x) e_{\alpha \beta}^{P}(x) d v=e_{\alpha \beta_{a}}^{P} v_{n} \int_{v_{m}} G(x, \alpha \beta ; n, \alpha \beta) e_{\alpha \beta}^{p}(x) d v \\
& +e_{\xi \eta_{a}}^{P} v_{n} \int_{v_{m}} G(x, \alpha \beta ; n, \alpha \beta) e_{\alpha \beta}^{P}(x) d v \\
& =e_{\alpha \beta_{v}}^{p} v_{n} G(m, \alpha \beta ; n, \alpha \beta) e_{\alpha \beta_{n}}^{P} v_{n}+e_{\xi \eta_{m}}^{P} v_{m} G(m, \alpha \beta ; n, \xi \eta) e_{\alpha \beta_{m}}^{p} v_{m}
\end{aligned}
$$

where 


$$
G(m, \alpha \beta ; n, \alpha \beta)=\frac{\int_{v} G(x, \alpha \beta ; n, \alpha \beta) e_{\alpha \beta}^{P}(x) d v}{\int_{v_{n}} e_{\alpha \beta}^{P}(x) d v}
$$

Writing the resid"al resolved shear stress in the mth grid as the left hand side of (23) divided



$$
\tau_{\alpha \beta_{m}}^{R}=G(m, \alpha \beta ; n, \alpha \beta) e_{\alpha \beta_{0}}^{p} v_{n}+G(m, \alpha \beta ; n, \bar{\zeta} \eta) e_{\xi \eta_{n}}^{p} v_{n}
$$

Letting the region I be the nth grid and region II, the mth grid, the left hand side of (23) becomes the left hand side of (12). Hence the reciprocal relation holds. Similarly we can write

$$
\tau_{\xi \eta_{m}}^{R}=G(m, \xi \eta ; n, \alpha \beta) e_{\alpha \beta_{0}}^{P} v_{n}+G(m . \xi \eta ; n, \xi \eta) e_{\xi \eta_{0}}^{p} v_{n}
$$

$G(m, \alpha \beta ; n, \alpha \beta)$ and similar terms are the influence coefficients for residual stresses caused by inelastic strains in different grids. Eq. (16) gives the reciprocal relation of these influence coefficients. If the plastic strain is taken to be constant in each grid, Eq. (22) reduces to

$$
G(x, \alpha \beta ; n, \alpha \beta)=\frac{1}{v_{n}} \int_{v_{3}} G(x, \alpha \beta ; \bar{x}, \alpha \beta) d v
$$

and Fig. (24) reduces to

$$
G(m, \alpha \beta ; n, \alpha \beta)=\frac{1}{v_{m}} \int_{v_{m}} G(x, \alpha \beta ; n, \alpha \beta) d v
$$

If there are $M$ grids with incremental $\Delta e_{j \eta}^{P}$ and $N$-grids with $\Delta e_{\alpha \beta}^{P}$. The residual stresses are written as

$$
\Delta \tau_{\alpha \beta_{m}}^{R}=\sum_{n=1}^{M} G(m, \alpha \beta ; n ., \xi \eta) \Delta e_{5 \eta}^{P} v_{n}+\sum_{n=1}^{N} G(m, \alpha \beta ; n, \alpha \beta) \Delta e_{\alpha \beta_{n}}^{P} v_{n}
$$




$$
\Delta \tau_{\xi \eta_{m}}^{R}=\sum_{n=1}^{M} G(m, \xi \eta ; n, \xi \eta) \Delta e_{\xi \eta}^{P} v_{n}+\sum_{n=1}^{N} G(m, \xi \eta ; n, \alpha \beta) \Delta e_{\alpha \beta_{n}}^{P} v_{n}
$$

In firding the incremental plastic strains in different grids, solution of a system of simultaneous equations is required. Due to the reciprocal relation, the matrix of these equations become symmetrical. This helps greatly the numerical solution.

Acknowledgement: The authors wish to acknowledge the support of the office of Naval Research under ONR contract N00014-86-K0153 and wish to acknowledge the support and encouragement of Dr. Yapa Rajapakse, ONR during the course of this study. 


\section{REFERENCES}

1. Lin. T.H., 1988. "Theory of Inelastic Structures," John Wiley and Sons, pp. 43-55.

2. Lin. T.H.. 1977, "Micromechanics of Slip Bands under Motions and Cyclic Loadings," Rev. Deformation Behavior of Materials: Edited by P. Feltham, Friend Publishing House, Israel pp. 264-314.

3. Lin. T.H. and Ito, Y.M., 1969, "Mechanics of Fatigue Crack Nucleation Mechanism," J. Mech. Phys. Solids, Vol. 17., pp. 511-503

4. Lin, S.R. and Lin, T.H., 1974, "Effects of Secondary Slip System on Early Fatigue Damage," J. Mech. Phys. Solids, Vol. 22., pp. 177-192

5. Lin, S.R. and Lin, T.H., 1983, "Initial Strain Fields and Fatigue Crack Initiation Mechanics" J. Appl. Mech. Vol. 105.

6. Taylor, G.1., 1938. "Plastic Strain in Metals" J. Inst. Metals Vol. 62., pp. 306-324. 